\title{
A construction of pseudorandom binary sequences using both additive and multiplicative characters
}

by

\section{LÁSZLÓ MÉRAI (Budapest)}

1. Introduction. In order to study the pseudorandomness of finite binary sequences, Mauduit and Sárközy introduced several definitions in [6]. For a given binary sequence

$$
E_{N}=\left\{e_{1}, \ldots, e_{N}\right\} \in\{-1,+1\}^{N}
$$

the well-distribution measure of $E_{N}$ is defined by

$$
W\left(E_{N}\right)=\max _{a, b, t}\left|U\left(E_{N}, t, a, b\right)\right|=\max _{a, b, t}\left|\sum_{j=0}^{t-1} e_{a+j b}\right|,
$$

where the maximum is taken over all $a, b, t \in \mathbb{N}$ such that $1 \leq a \leq a+$ $(t-1) b \leq N$, and the correlation measure of order $l$ of $E_{N}$ is defined as

$$
C_{l}\left(E_{N}\right)=\max _{M, D}\left|V\left(E_{N}, M, D\right)\right|=\max _{M, D}\left|\sum_{n=1}^{M} e_{n+d_{1}} \ldots e_{n+d_{l}}\right|,
$$

where the maximum is taken over all $D=\left(d_{1}, \ldots, d_{l}\right)$ and $M$ such that $0 \leq d_{1}<\cdots<d_{l} \leq N-M$.

The sequence $E_{N}$ is considered to be a "good" pseudorandom sequence if both these measures $W\left(E_{N}\right)$ and $C_{l}\left(E_{N}\right)$ (at least for small $l$ ) are "small" in terms of $N$ (in particular, both are $o(N)$ as $N \rightarrow \infty$ ). This terminology is justified since for a truly random sequence $E_{N}$ each of these measures is $\ll \sqrt{N \log N}$. (For a more precise version of this result see [1].)

Using the Legendre symbol, Mauduit and Sárközy [6] showed an example of a "good" pseudorandom sequence. They defined a binary sequence by putting $N=p-1$ where $p$ is a prime number, and

$$
e_{n}=\left(\frac{n}{p}\right) \quad \text { for } n=1, \ldots, p-1 .
$$

2000 Mathematics Subject Classification: Primary 11K45.

Key words and phrases: pseudorandom, binary sequence, hybrid character sum, rational function. 
They proved that

$$
W\left(E_{p-1}\right) \ll p^{1 / 2} \log p, \quad C_{l}\left(E_{p-1}\right) \ll l p^{1 / 2} \log p .
$$

Other large families of binary sequences with strong pseudorandom properties were studied in [4], [3], [5], [8], [7], [10].

In this paper a new construction of a large family of pseudorandom binary sequences is presented which uses both additive and multiplicative characters.

Let $p$ be a prime, $\psi$ an additive character, $\chi$ a multiplicative character in $\mathbb{F}_{p}, \alpha \in \mathbb{C}$ with $|\alpha|=1$, and $f(x), g(x), q(x), r(x) \in \mathbb{F}_{p}[x]$. Let us define $E_{p}$ by

$$
e_{n}= \begin{cases}+1 \quad \text { if } \mathfrak{R e}\left(\alpha \psi\left(\frac{f(n)}{g(n)}\right) \chi\left(\frac{q(n)}{r(n)}\right)\right) \geq 0 \\ \text { and } g(n), r(n), q(n) \neq 0, \\ -1 \quad \text { otherwise. }\end{cases}
$$

Note that this construction generalizes several earlier ones:

Construction 1: If $\chi$ is the Legendre symbol, $\psi$ is the trivial additive character, $\alpha=1, r(x)$ is a non-zero constant polynomial, we get an extended variant of (1), studied in [3]:

$$
e_{n}=\left\{\begin{array}{ll}
\left(\frac{q(n)}{p}\right) & \text { for } p \nmid q(n), \\
1 & \text { for } p \mid q(n),
\end{array} \quad \text { for } n=1, \ldots, p .\right.
$$

Construction 2: If $\chi$ is a general multiplicative character, $\psi$ is the trivial additive character, $\alpha=1, r(x)$ is a non-zero constant polynomial, we get the construction studied in [8], [10], [9]:

$$
e_{n}=\left\{\begin{array}{ll}
+1 & \text { if } \mathfrak{R e}(\chi(q(n))) \geq 0, \\
-1 & \text { otherwise, }
\end{array} \quad \text { for } n=1, \ldots, p .\right.
$$

Construction 3: If $\psi$ is the additive character of the form $\psi(n)=$ $e(n / p)$ (where now $\left.e(\alpha)=e^{2 \pi i \alpha}\right), \chi$ is the trivial multiplicative character, $\alpha=i$, then we get a variant of pseudorandom sequences studied in [4], [5], [7]:

$$
e_{n}= \begin{cases}+1 & \text { if } r_{p}\left(\frac{f(n)}{g(n)}\right)<\frac{p}{2} \text { for } p \nmid g(n), \quad \text { for } n=1, \ldots, p, \\ -1 & \text { otherwise },\end{cases}
$$

where $r_{p}(n)$ denotes the least non-negative residue of $n$ modulo $p$.

Let us introduce the following notations: for a rational function $F(x)=$ $f(x) / g(x)$ let $\operatorname{deg} F(x)=\operatorname{deg} f(x)-\operatorname{deg} g(x)$ and $\operatorname{deg}^{*} F(x)=\operatorname{deg} f(x)+$ $\operatorname{deg} g(x)$. Finally, let us denote the algebraic closure of $\mathbb{F}_{p}$ by $\overline{\mathbb{F}}_{p}$. 
TheOREM 1. Assume that $p$ is a prime number, $\chi$ is a non-principal multiplicative character modulo $p$ of order $d, \psi$ is a non-principal additive character modulo $p, \alpha \in \mathbb{C}$ with $|\alpha|=1, F(x)=f(x) / g(x), Q(x)=$ $q(x) / r(x) \in \mathbb{F}_{p}(x)$ are rational functions such that $(g(x), f(x))=1$ and $(q(x), r(x))=1$ and neither $q(x)$ nor $r(x)$ has a multiple zero in $\overline{\mathbb{F}}_{p}$, and the binary sequence $E_{p}=\left\{e_{1}, \ldots, e_{p}\right\}$ is defined by (2). Then

$$
W\left(E_{p}\right) \ll\left(\operatorname{deg}^{*} F+d \operatorname{deg}^{*} Q\right) p^{1 / 2}(\log p)^{2} .
$$

Theorem 2. Let $p, F(x), Q(x)$ and $E_{p}$ be as in Theorem 1. Assume also that $l \in \mathbb{N}, 2 \leq l<p$ and one of the following conditions holds:

(a) $l=2$;

(b) $(4 \operatorname{deg} g)^{l}<p,\left(4 \operatorname{deg}^{*} Q\right)^{l}<p$;

(c) $g(x)=\left(x+a_{1}\right) \ldots\left(x+a_{k}\right)\left(\right.$ with $a_{i} \neq a_{j}$ for $\left.i \neq j\right)$ and $l \operatorname{deg} g<p / 2$, $\left(4 \text { deg* }^{*}\right)^{l}<p$.

Then

$$
C_{l}\left(E_{p}\right) \ll(l+1)\left(\operatorname{deg}^{*} F+d \operatorname{deg}^{*} Q\right) p^{1 / 2}(\log p)^{l+1} .
$$

2. On hybrid character sums. The proofs of Theorems 1 and 2 will be based on hybrid character sum estimates. For rational functions $F(x), Q(x)$ $\in \mathbb{F}_{p}(x)$ denote the union of the sets of poles of $F(x)$ and $Q(x)$ by $\mathcal{S}$.

Definition 3. For $F(x), Q(x) \in \mathbb{F}_{q}(x)$ the character sum

is degenerate if

$$
\sum_{n \notin \mathcal{S}} \psi(F(n)) \chi(Q(n))
$$

$$
F(x)=H(x)^{p}-H(x)+b \quad \text { for some } b \in \mathbb{F}_{q} \text { and } H(x) \in \mathbb{F}_{q}(x)
$$

and

$$
Q(x)=b H(x)^{d} \quad \text { for some } b \in \mathbb{F}_{q} \text { and } H(x) \in \mathbb{F}_{q}(x) .
$$

If the character sum is degenerate, then all of the terms are constant, so one cannot give a non-trivial upper bound for the sum. For non-degenerate sums Perel'muter gave a non-trivial upper bound in [11]:

TheOREM 4. Let $\mathbb{F}_{q}$ be a finite field of characteristic $p, \chi$ be a nonprincipal multiplicative character of $\mathbb{F}_{q}$ of order $d$, and $\psi$ be a non-principal additive character of $\mathbb{F}_{q}$. Let $F(x)=f(x) / g(x), Q(x)=q(x) / r(x) \in \mathbb{F}_{q}(x)$. Assume that the hybrid character sum is not degenerate and the following conditions hold:

(1) If $F=f / g_{1}^{\lambda_{1}} \ldots g_{r}^{\lambda_{r}}$, where the polynomials $g_{1}, \ldots, g_{r}$ are non-constants and $\left(g_{1}, \ldots, g_{r}\right)=1$ then $p \nmid \lambda_{i}$ when $\lambda_{i}>0$ for $i=1, \ldots, r$ and $p \nmid \operatorname{deg} F$ when $\operatorname{deg} F>0$.

(2) If $Q=q_{1}^{n_{1}} \ldots q_{u}^{n_{u}} / r_{1}^{m_{1}} \ldots r_{v}^{m_{v}}$ then $0<n_{i}, m_{i}<d$ for all $i$. 
Then

$$
\left|\sum_{n \notin \mathcal{S}} \psi(F(n)) \chi(Q(n))\right| \leq\left(d_{1}+d_{2}-2\right) q^{1 / 2}+d_{1}+d_{2}+1
$$

with

$$
d_{1}=\max \{\operatorname{deg} f, \operatorname{deg} g\}+s+\lambda, \quad d_{2}=\operatorname{deg} q+\operatorname{deg} r+\mu,
$$

where $s$ is the number of distinct zeros of $g, \lambda$ is 0 if $\operatorname{deg} g \geq \operatorname{deg} f$ and 1 otherwise, $\mu$ is 0 if $d \mid \operatorname{deg} Q$ and 1 otherwise.

THEOREM 5. Let $p$ be a prime, let $\psi$ be a non-principal additive character of $\mathbb{F}_{p}$, and $\chi$ a non-principal multiplicative character of $\mathbb{F}_{p}$ of order $d$. Furthermore, let $F=f / g, Q=q / r$ be non-zero rational functions over $\mathbb{F}_{p}$, and let $s$ be the number of distinct zeros of $g$ in $\overline{\mathbb{F}}_{p}$. Suppose that $g(x) \nmid f(x)$ and $Q(x)$ is not of the form $b B(x)^{d}$ for any $b \in \mathbb{F}_{p}$ and $B(x) \in \mathbb{F}_{p}(x)$. If $1 \leq N<p$ then

$$
\begin{aligned}
& \left|\sum_{\substack{0 \leq n<N \\
n \notin \mathcal{S}}} \psi(F(n)) \chi(Q(n))\right| \\
& \quad \leq 3(\max \{\operatorname{deg} f, \operatorname{deg} g\}+s+\operatorname{deg} q+\operatorname{deg} r) p^{1 / 2} \log p .
\end{aligned}
$$

Proof. We can assume that the degrees of all the polynomials are less than $p$ since the result is trivial otherwise.

It follows from the basic properties of additive characters that

$$
\sum_{r=0}^{N-1} \frac{1}{p} \sum_{u=0}^{p-1} \psi(u(n-r))= \begin{cases}1 & \text { if } 0 \leq n<N \\ 0 & \text { otherwise }\end{cases}
$$

Let us denote the character sum in (6) by $S_{N}$. We have

$$
\begin{aligned}
S_{N}= & \sum_{n \notin \mathcal{S}} \psi(F(n)) \chi(Q(n)) \sum_{r=0}^{N-1} \frac{1}{p} \sum_{u=0}^{p-1} \psi(u(n-r)) \\
= & \frac{1}{p} \sum_{u=0}^{p-1}\left(\sum_{r=0}^{N-1} \psi(-u r)\right)\left(\sum_{n \notin \mathcal{S}} \psi(F(n)+u n) \chi(Q(n))\right) \\
= & \frac{1}{p} \sum_{u=1}^{p-1}\left(\sum_{r=0}^{N-1} \psi(-u r)\right)\left(\sum_{n \notin \mathcal{S}} \psi(F(n)+u n) \chi(Q(n))\right) \\
& +\frac{N}{p} \sum_{n \notin \mathcal{S}} \psi(F(n)) \chi(Q(n))
\end{aligned}
$$


and so

$$
\begin{aligned}
\left|S_{N}\right| \leq & \frac{1}{p} \sum_{u=1}^{p-1}\left|\sum_{r=0}^{N-1} \psi(u r)\right|\left|\sum_{n \notin \mathcal{S}} \psi(F(n)+u n) \chi(Q(n))\right| \\
& +\frac{N}{p}\left|\sum_{n \notin \mathcal{S}} \psi(F(n)) \chi(Q(n))\right| .
\end{aligned}
$$

For a fixed $u$ we consider the rational function

$$
F_{u}(x)=F(x)+u x=\frac{f(x)}{g(x)}+u x .
$$

To show that $F_{u}(x)$ satisfies the conditions of Theorem 4 , it suffices to prove that $F_{u}(x)$ is not of the form $A(x)^{p}-A(x)$ with $A(x) \in \overline{\mathbb{F}}_{p}(x)$. Suppose that

$$
F_{u}(x)=\left(\frac{K(x)}{L(x)}\right)^{p}-\frac{K(x)}{L(x)}
$$

with $K(x), L(x) \in \overline{\mathbb{F}}_{p}[x]$ such that $(K(x), L(x))=1$. Then

$$
L(x)^{p}(f(x)+u x g(x))=\left(K(x)^{p-1}-L(x)^{p-1}\right) K(x) g(x),
$$

so $L(x)^{p} \mid g(x)$ as $(K(x), L(x))=1$. Since $\operatorname{deg} g(x)<p$, it follows that $L(x)$ is a nonzero constant polynomial. Thus we get

$$
f(x)+u x g(x)=\left(\alpha K(x)^{p}+\beta K(x)\right) g(x),
$$

and hence

$$
f(x)=\left(\alpha K(x)^{p}+\beta K(x)-u x\right) g(x),
$$

for some $\alpha, \beta \in \overline{\mathbb{F}}_{p}$ with $\alpha \beta \neq 0$.

Since $g(x) \nmid f(x)$ and either

$$
\operatorname{deg}\left(\alpha K(x)^{p}+\beta K(x)-u x\right)>p
$$

or

$$
\operatorname{deg}\left(\alpha K(x)^{p}+\beta K(x)-u x\right)=1
$$

we see that (8) cannot hold.

Since $F(x)+u x, F(x)$ and $Q(x)$ satisfy the conditions of Theorem 4 , we deduce from $(7)$ that

$$
\begin{aligned}
\left|S_{N}\right| \leq & \frac{1}{p}\left(\sum_{u=1}^{p-1}\left|\sum_{r=0}^{N-1} \psi(u r)\right|+N\right) \\
& \cdot 2(\max \{\operatorname{deg} f, \operatorname{deg} g\}+s+\operatorname{deg} q+\operatorname{deg} r) p^{1 / 2}
\end{aligned}
$$

and

$$
\sum_{u=0}^{p-1}\left|\sum_{r=0}^{N-1} \psi(u r)\right|<\frac{4}{\pi} p \log p+0.38 p+0.64
$$

by Theorem 1 in [2]. 
3. The well-distribution measure. To express the terms of $E_{p}$, we will need the generalization of Lemma 2 in [4].

Lemma 6. Let $m \in \mathbb{N}$, and let $\varepsilon$ be an $m$ th root of unity. Then

$$
\frac{1}{m} \sum_{-[m / 2]<a \leq[m / 2]} v_{m}(a) \varepsilon^{a}= \begin{cases}+1 & \text { if }-\pi / 2 \leq \arg (\varepsilon)<\pi / 2, \\ -1 & \text { otherwise }\end{cases}
$$

where $v_{m}(a)$ is a function of period $m$ such that $v_{m}(0)=1$, and if $m$ is odd, then

$$
v_{m}(a)=i^{a}\left(1+i \frac{(-1)^{a}-\cos (\pi a / m)}{\sin (\pi a / m)}\right) \quad \text { if } 1 \leq|a|<m / 2,
$$

while if $m$ is even, then

$$
v_{m}(a)=\left\{\begin{array}{ll}
0 & \text { if } a \text { is even } \\
i^{a}\left(2-2 i \frac{\cos (a \pi / m)}{\sin (a \pi / m)}\right) & \text { if } a \text { is odd }
\end{array} \quad \text { if } 1 \leq|a| \leq m / 2 .\right.
$$

Furthermore, in both cases, $v_{m}(a) \ll m / a$ if $a \neq 0$.

Proof. For $m$ odd, the statement has been proved in [4]; for $m$ even the proof is similar.

Proof of Theorem 1. To prove the desired inequality, consider $a \in \mathbb{Z}$ and $b, t \in \mathbb{N}$ such that

$$
1 \leq a \leq a+(t-1) b \leq p, \quad b<p .
$$

Then by Lemma 6 we have

$$
\begin{aligned}
U & \left(E_{p}, t, a, b\right)=\sum_{j=0}^{t-1} e_{a+j b} \\
= & \frac{1}{d p} \sum_{-[d p / 2]<h \leq[d p / 2]} v_{d p}(h) \alpha^{h} \\
& \cdot\left(\sum_{\substack{0 \leq j \leq t-1 \\
a+j b \notin \mathcal{S}}} \psi(F(a+j b))^{h} \chi(Q(a+j b))^{h}+\mathcal{O}\left(\sum_{\substack{0 \leq j \leq p \\
a+j b \in \mathcal{S}}} 1\right)\right)+\mathcal{O}(\operatorname{deg} f) \\
= & \frac{1}{d p} \sum_{-[d p / 2]<h \leq[d p / 2]} v_{d p}(h) \alpha^{h}\left(\sum_{\substack{0 \leq j \leq t-1 \\
a+j b \notin \mathcal{S}}} \psi(F(a+j b))^{h} \chi(Q(a+j b))^{r_{d}(h)}\right) \\
& +\mathcal{O}(|\mathcal{S}|)+\mathcal{O}(\operatorname{deg} f),
\end{aligned}
$$

since $\chi(Q(n))^{h}=\chi(Q(n))^{r_{d}(h)}$ for $n \in \mathbb{F}_{p}$. 
If $0<|h| \leq d p / 2$ then $h \nmid p$ or $h \nmid d$ (and so $r_{d}(h) \nmid d$ ), thus the hybrid character sums are not degenerate. Furthermore,

$$
\max \{\operatorname{deg} f, \operatorname{deg} g\}+s \leq 2(\operatorname{deg} f+\operatorname{deg} g)
$$

and

$$
\operatorname{deg}^{*} Q^{r_{d}(h)}=r_{d}(h) \operatorname{deg}^{*} Q \leq d \operatorname{deg}^{*} Q,
$$

thus by Theorem 5 we have

$$
\begin{aligned}
& \left|U\left(E_{p}, t, a, b\right)\right|=\left|\sum_{j=0}^{t-1} e_{a+j b}\right| \\
& \leq \frac{1}{d p} \sum_{\substack{-[d p / 2]<h \leq[d p / 2] \\
h \neq 0}}\left|v_{d p}(h)\right| \sum_{\substack{0 \leq j \leq t-1 \\
a+j b \notin \mathcal{S}}} \psi(F(a+j b))^{h} \chi(Q(a+j b))^{r_{d}(h) \mid} \\
& \quad+\left|v_{d p}(0)\right|+\mathcal{O}(|\mathcal{S}|)+\mathcal{O}(\operatorname{deg} f) \\
& \ll \\
& \quad \frac{1}{d p} \sum_{-[d p / 2]<h \leq[d p / 2]}\left|v_{d p}(h)\right|\left(\operatorname{deg}^{*} F+\operatorname{deg}^{*} Q^{r_{d}(h)}\right) p^{1 / 2} \log p+\left|v_{d p}(0)\right| \\
& \ll\left(\operatorname{deg}^{*} F+\operatorname{deg}^{*} Q^{r_{d}(h)}\right) p^{1 / 2} \log p \sum_{\substack{-[d p / 2]<h \leq[d p / 2] \\
h \neq 0}} \frac{1}{|h|} \\
& \ll\left(\operatorname{deg}^{*} F+d \operatorname{deg}^{*} Q\right) p^{1 / 2}(\log p)^{2} . \text { - }
\end{aligned}
$$

\section{The correlation measure}

Proof of Theorem 2. Consider any $M<p$ and $D=\left(d_{1}, \ldots, d_{l}\right)$ such that $0 \leq d_{1}<\cdots<d_{l} \leq p-M$. Then

$$
\begin{aligned}
V\left(E_{p}, M, D\right)= & \sum_{n=1}^{M} e_{n+d_{1}} \ldots e_{n+d_{l}} \\
= & \frac{1}{(d p)^{l}} \sum_{\substack{1 \leq n \leq M \\
n+d_{1}, \ldots, n+d_{l} \notin \mathcal{S}}} \prod_{\substack{i=1 \\
-}}^{l} \sum_{-[d p / 2]<h_{i} \leq[d p / 2]} v_{d p}\left(h_{i}\right) \\
& \cdot \alpha^{h_{i}}\left(\psi\left(F\left(n+d_{i}\right)\right) \chi\left(Q\left(n+d_{i}\right)\right)\right)^{h_{i}} \\
& +\mathcal{O}\left(\sum_{\substack{1 \leq n \leq M \\
n+d_{1} \in \mathcal{S}}} 1+\cdots+\sum_{\substack{1 \leq n \leq M \\
n+d_{l} \in \mathcal{S}}} 1\right)+\mathcal{O}(l \operatorname{deg} f),
\end{aligned}
$$

whence, separating the contribution of the term with $h_{1}=\cdots=h_{l}=0$, 
(10)

$$
\begin{aligned}
& V\left(E_{p}, M, D\right)=\frac{1}{(d p)^{l}}(M+\mathcal{O}(|\mathcal{S}| l)) \\
& +\frac{1}{(d p)^{l}} \sum_{\substack{-[d p / 2]<h_{1} \leq[d p / 2] \\
\left(h_{1}, \ldots, h_{l}\right) \neq(0, \ldots, 0)}} \ldots \sum_{-[d p / 2]<h_{l} \leq[d p / 2]} v_{d p}\left(h_{1}\right) \ldots v_{d p}\left(h_{l}\right) \prod_{i=1}^{l} \alpha^{h_{i}} \\
& \quad \sum_{\substack{1 \leq n \leq M \\
n+d_{1}, \ldots, n+d_{l} \notin \mathcal{S}}} \prod_{i=1}^{l}\left(\psi\left(F\left(n+d_{i}\right)\right) \chi\left(Q\left(n+d_{i}\right)\right)\right)^{h_{i}} \\
& +\mathcal{O}(|\mathcal{S}| l)+\mathcal{O}(l \operatorname{deg} f) .
\end{aligned}
$$

Now consider one of the innermost sums (where $\left.\left(h_{1}, \ldots, h_{l}\right) \neq(0, \ldots, 0)\right)$, and let $h_{i_{1}}<\cdots<h_{i_{r}}$ be the non-zero $h_{i}$ 's. Then

$$
\begin{aligned}
& \sum_{\substack{1 \leq n \leq M \\
n+d_{1}, \ldots, n+d_{l} \notin \mathcal{S}}} \prod_{i=1}^{l}\left(\psi\left(F\left(n+d_{i}\right)\right) \chi\left(Q\left(n+d_{i}\right)\right)\right)^{h_{i}} \\
= & \sum_{\substack{1 \leq n \leq M \\
n+d_{1}, \ldots, n+d_{l} \notin \mathcal{S}}} \psi\left(\sum_{i=1}^{l} h_{i} F\left(n+d_{i}\right)\right) \chi\left(\prod_{i=1}^{l} Q\left(n+d_{i}\right)^{h_{i}}\right) \\
= & \sum_{\substack{1 \leq n \leq M \\
n+d_{i_{1}}, \ldots, n+d_{i_{r}} \notin \mathcal{S}}} \psi\left(\sum_{j=1}^{r} h_{i_{j}} F\left(n+d_{i_{j}}\right)\right) \chi\left(\prod_{j=1}^{r} Q\left(n+d_{i_{j}}\right)^{r_{d}\left(h_{i_{j}}\right)}\right) \\
= & \sum_{\substack{1 \leq n \leq M \\
n+d_{i_{1}}, \ldots, n+d_{i_{r}} \notin \mathcal{S}}} \psi\left(\frac{f_{h_{1}, \ldots, h_{l}}(n)}{g_{h_{1}, \ldots, h_{l}}(n)}\right) \chi\left(\frac{q_{h_{1}, \ldots, h_{l}}(n)}{r_{h_{1}, \ldots, h_{l}}(n)}\right)
\end{aligned}
$$

with

$$
\begin{aligned}
f_{h_{1}, \ldots, h_{l}}(x) & =\sum_{t=1}^{r} h_{i_{t}} f\left(x+d_{i_{t}}\right) \prod_{\substack{1 \leq j \leq r \\
j \neq t}} g\left(x+d_{i_{j}}\right), \\
g_{h_{1}, \ldots, h_{l}}(x) & =\prod_{j=1}^{r} g\left(x+d_{i_{j}}\right), \\
q_{h_{1}, \ldots, h_{l}}(x) & =\prod_{j=1}^{r} q\left(x+d_{i_{j}}\right)^{r_{d}\left(h_{i_{j}}\right)} \\
r_{h_{1}, \ldots, h_{l}}(x) & =\prod_{j=1}^{r} r\left(x+d_{i_{j}}\right)^{r_{d}\left(h_{i_{j}}\right)}
\end{aligned}
$$


so that

$$
\begin{aligned}
\operatorname{deg} f_{h_{1}, \ldots, h_{l}} & \leq \operatorname{deg} f+(r-1) \operatorname{deg} g \leq \operatorname{deg} f+(l-1) \operatorname{deg} g, \\
\operatorname{deg} g_{h_{1}, \ldots, h_{l}} & =r \operatorname{deg} g \leq l \operatorname{deg} g \\
\operatorname{deg}^{*}\left(\frac{q_{h_{1}, \ldots, h_{l}}}{r_{h_{1}, \ldots, h_{l}}}\right) & \leq \sum_{j=1}^{r} r_{d}\left(h_{i_{j}}\right) \operatorname{deg}^{*} Q \leq l d \operatorname{deg}^{*} Q .
\end{aligned}
$$

In order to give an upper bound for the character sum in (11), we have to show that this sum is not degenerate for every $\left(h_{1}, \ldots, h_{l}\right) \neq(0, \ldots, 0)$.

First, suppose that $p \nmid h_{i_{j}}$ for all $j=1, \ldots, r$. The following lemma (Lemmas 8 and 9 in [7]) shows that the character sum is not degenerate.

LEMmA 7. If $p, f(x), g(x)$ and $l$ satisfy the conditions in Theorem 2 and $p \nmid h_{i_{j}}$ for $j=1, \ldots, r$, then $g_{h_{1}, \ldots, h_{l}}(x) \nmid f_{h_{1}, \ldots, h_{l}}(x)$.

By the lemma, from (11) we have

$$
\begin{gathered}
\left|\sum_{\substack{1 \leq n \leq M \\
n+d_{i_{1}}, \ldots, n+d_{i_{r}} \notin \mathcal{S}}} \psi\left(\frac{f_{h_{1}, \ldots, h_{l}}(n)}{g_{h_{1}, \ldots, h_{l}}(n)}\right) \chi\left(\frac{q_{h_{1}, \ldots, h_{l}}(n)}{r_{h_{1}, \ldots, h_{l}}(n)}\right)\right| \\
\leq 3\left(\operatorname{deg}^{*}\left(\frac{f_{h_{1}, \ldots, h_{l}}}{g_{h_{1}, \ldots, h_{l}}}\right)+\operatorname{deg}^{*}\left(\frac{q_{h_{1}, \ldots, h_{l}}}{r_{h_{1}, \ldots, h_{l}}}\right)\right) p^{1 / 2} \log p \\
\leq 3(l+1)\left(\operatorname{deg}^{*} F+d \operatorname{deg}^{*} Q\right) p^{1 / 2} \log p,
\end{gathered}
$$

since

$$
\begin{aligned}
\max \left\{\operatorname{deg} f_{h_{1}, \ldots, h_{l}}, \operatorname{deg} g_{h_{1}, \ldots, h_{l}}\right\}+s_{h_{1}, \ldots, h_{l}} & \leq \operatorname{deg} f+(l+1) \operatorname{deg} g \\
& \leq(l+1) \operatorname{deg}^{*} F
\end{aligned}
$$

where $s_{h_{1}, \ldots, h_{l}}$ is the number of distinct zeros of $g_{h_{1}, \ldots, h_{l}}$.

On the other hand, if there are some $h_{i_{j}}$ such that $p \mid h_{i_{j}}$, then $d \nmid h_{i_{j}}$ since $0<\left|h_{i_{j}}\right| \leq[d p / 2]$. Let

$$
q_{h_{1}, \ldots, h_{l}}^{\prime}(x)=\prod_{\substack{j=1 \\ d \nmid h_{i_{j}}}}^{r} q\left(x+d_{i_{j}}\right)^{r_{d}\left(h_{i_{j}}\right)}, \quad r_{h_{1}, \ldots, h_{l}}^{\prime}(x)=\prod_{\substack{j=1 \\ d \nmid h_{i_{j}}}}^{r} r\left(x+d_{i_{j}}\right)^{r_{d}\left(h_{i_{j}}\right)} .
$$

From the assumption, none of these polynomials is constant. Thus it is enough to prove the following lemma:

LEMMA 8. If $p, q(x), r(x)$ and $l$ satisfy the conditions in Theorem 2 and there exists an index $j$ such that $d \nmid h_{i_{j}}$, then

$$
\frac{q_{h_{1}, \ldots, h_{l}}^{\prime}(x)}{r_{h_{1}, \ldots, h_{l}}^{\prime}(x)}=b B(x)^{d}
$$

for no $b \in \mathbb{F}_{p}$ and $B(x) \in \mathbb{F}_{p}(x)$. 
In order to prove this, we will need the following lemma from [5].

Lemma 9. Assume that $p$ is a prime number, $k, l \in \mathbb{N}$ and $k, l<p$. Assume also that one of the following conditions holds:

(1) $l \leq 2$,

(2) $(4 k)^{l}<p$.

Then for all $\mathcal{A}, \mathcal{B} \subset \mathbb{Z}_{p}$ with $|\mathcal{A}|=k$ and $|\mathcal{B}|=l$, there is a $c \in \mathbb{Z}_{p}$ such that the equation

$$
a+b=c, \quad a \in \mathcal{A}, b \in \mathcal{B},
$$

has exactly one solution in $a, b$.

Proof of Lemma 8. We use the approach developed in [3]. We say that $\varrho(x), \sigma(x) \in \mathbb{F}_{p}[x]$ are equivalent, $\sigma \sim \varrho$, if there is an $a \in \mathbb{F}_{p}$ such that $\varrho(x+a)=\sigma(x)$. Clearly, this is an equivalence relation.

Write $q(x)$ and $r(x)$ as the product of irreducible polynomials over $\mathbb{F}_{p}$. It follows from our assumption on the polynomials that all of these irreducible factors are distinct. Let us divide these factors into groups of equivalent factors. A typical group has the following form: $\varrho\left(x+a_{1}\right), \ldots, \varrho\left(x+a_{u}\right)$ (where $u \leq \operatorname{deg} q$ ) belong to $q(x)$, and $\varrho\left(x+b_{1}\right), \ldots, \varrho\left(x+b_{v}\right)$ (where $v \leq \operatorname{deg} r$ ) belong to $r(x)$, where the constants $a_{i}, b_{j}$ are distinct by assumption.

By the definition of $q_{h_{1}, \ldots, h_{l}}^{\prime}$ and $r_{h_{1}, \ldots, h_{l}}^{\prime}$ the factors occurring in the polynomials for a given group have the following form: $\varrho\left(x+a_{t}+d_{i_{j}}\right)$ for $t=1, \ldots, u$ and $j=1, \ldots, r$ and $\varrho\left(x+b_{z}+d_{i_{j}}\right)$ resp. All these polynomials are equivalent, and no other irreducible factor belongs to this equivalence class.

Now set $\mathcal{A}=\left\{a_{1}, \ldots, a_{u}, b_{1}, \ldots, b_{v}\right\}, \mathcal{B}=\left\{d_{i_{1}}, \ldots, d_{i_{r}}\right\}$. It follows from assumption of Theorem 2 that either

$$
|\mathcal{B}|=r \leq l=2
$$

or

$$
(4|\mathcal{A}|)^{|\mathcal{B}|} \leq(4(\operatorname{deg} q+\operatorname{deg} r))^{l} \leq\left(4 \operatorname{deg}^{*} Q\right)^{l}<p,
$$

so that one of the assumptions (1) or (2) in Lemma 9 holds, and thus the lemma can be applied. Hence there is a $c \in \mathbb{F}_{p}$ that has exactly one representation (13). Thus either $\varrho(x+c) \nmid q_{h_{1}, \ldots, h_{l}}^{\prime}(x)$ or $\varrho(x+c) \nmid r_{h_{1}, \ldots, h_{l}}^{\prime}(x)$, so

$$
\varrho(x+c) \mid q_{h_{1}, \ldots, h_{l}}^{\prime}(x)\left(r_{h_{1}, \ldots, h_{l}}^{\prime}(x)\right)^{d-1}
$$

but

$$
(\varrho(x+c))^{d} \nmid q_{h_{1}, \ldots, h_{l}}^{\prime}(x)\left(r_{h_{1}, \ldots, h_{l}}^{\prime}(x)\right)^{d-1} .
$$

By Lemma 8 the character sum in (12) is not degenerate, so the inequality also holds if there are some $h_{i_{j}}$ such that $p \mid h_{i_{j}}$. 
Thus (10) and (12) yield

$$
\begin{aligned}
& \left|V\left(E_{p}, M, D\right)\right| \\
& \ll \frac{1}{(d p)^{l}}\left|\sum_{-[d p / 2]<h_{1} \leq[d p / 2]} \ldots \sum_{\substack{-[d p / 2]<h_{l} \leq[d p / 2] \\
\left(h_{1}, \ldots, h_{l}\right) \neq(0, \ldots, 0)}} v_{d p}\left(h_{1}\right) \ldots v_{d p}\left(h_{l}\right)\right| \\
& \cdot\left|\sum_{\substack{1 \leq n \leq M \\
n+d_{1}, \ldots, n+d_{l} \notin \mathcal{S}}} \psi\left(\prod_{i=1}^{l} h_{i} F\left(n+d_{i}\right)\right) \chi\left(\sum_{i=1}^{l} Q\left(n+d_{i}\right)^{h_{i}}\right)\right| \\
& +\mathcal{O}(|\mathcal{S}| l)+\mathcal{O}(l \operatorname{deg} f) \\
& \ll \frac{1}{(d p)^{l}}(l+1)\left(\operatorname{deg}^{*} F+d \operatorname{deg}^{*} Q\right) p^{1 / 2} \log p\left(\sum_{|h|<d p / 2}\left|v_{d p}(h)\right|\right)^{l} \\
& +\mathcal{O}(|\mathcal{S}| l)+\mathcal{O}(l \operatorname{deg} f) \\
& \ll \frac{1}{(d p)^{l}}(l+1)\left(\operatorname{deg}^{*} F+d \operatorname{deg}^{*} Q\right) p^{1 / 2} \log p\left(1+\sum_{0<|h|<d p / 2} \frac{d p}{h}\right)^{l} \\
& +\mathcal{O}(|\mathcal{S}| l)+\mathcal{O}\left(l \operatorname{deg}^{*} Q\right) \\
& \ll(l+1)\left(\operatorname{deg}^{*} F+d \operatorname{deg}^{*} Q\right) p^{1 / 2}(\log p)^{l+1},
\end{aligned}
$$

which completes the proof of Theorem 2 .

\section{References}

[1] N. Alon, Y. Kohayakawa, C. Mauduit, C. G. Moreira and V. Rödl, Measures of pseudorandomness for finite sequences: typical values, Proc. London Math. Soc. (3) 95 (2007), 778-812.

[2] T. Cochrane, On a trigonometric inequality of Vinogradov, J. Number Theory 27 (1987), 9-16.

[3] L. Goubin, C. Mauduit and A. Sárközy, Construction of large families of pseudorandom binary sequences, J. Number Theory 106 (2004), 56-69.

[4] C. Mauduit, J. Rivat and A. Sárközy, Construction of pseudorandom binary sequence using additive characters, Monatsh. Math. 141 (2004), 197-208.

[5] C. Mauduit and A. Sárközy, Construction of pseudorandom binary sequences by using the multiplicative inverse, Acta Math. Hungar. 108 (2005), 239-252.

[6] -, -, On finite pseudorandom binary sequences I: Measure of pseudorandomness, the Legendre symbol, Acta Arith. 82 (1997), 365-377.

[7] L. Mérai, A construction of pseudorandom binary sequences using rational functions, Uniform Distribution, to appear.

[8] -, Construction of large families of pseudorandom binary sequences, Ramanujan J., to appear.

[9] S. M. Oon, Construction des suites binaires pseudo-aléatoires, PhD thesis, Nancy, 2005. 
[10] S. M. Oon, On pseudo-random properties of certain Dirichlet series, Ramanujan J. 15 (2008), 19-30.

[11] G. I. Perel'muter, On certain character sums, Uspekhi Mat. Nauk 18 (1963), no. 2, 145-149.

Department of Algebra and Number Theory

Eötvös Loránd University

Pázmány Péter Sétány 1/c

1117 Budapest, Hungary

E-mail: merai@cs.elte.hu

Received on 18.8.2008

and in revised form on 13.2.2009 